\title{
Cultural Adaptation and Psychometric Testing of the Short Form of Iranian Childbirth Self Efficacy Inventory
}

\author{
Mahboubeh Khorsandi ${ }^{1}$, Mohammad Asghari Jafarabadi ${ }^{2,}{ }^{*}$, Farzaneh Jahani ${ }^{1}$, Moham- \\ mad Rafiei ${ }^{1}$ \\ ${ }^{1}$ Arak University of Medical Sciences, Arak, IR Iran \\ ${ }^{2}$ Medical Education Research Center, Faculty of Health, Tabriz University of Medical Sciences, Tabriz, IR Iran
}

* Corresponding Author: Mohammad Asghari Jafarabadi, Medical Education Research Center, Faculty of Health, Tabriz University of Medical Sciences, Tabriz, IR Iran. Tel: +984113357580-2(ext: 383), Fax:+98-4113340634, E-mail: m.asghari862@gmail.com

Received: April 23, 2013; Revised: August 13, 2013; Accepted: September 22, 2013

\begin{abstract}
Background:To assess maternal confidence in her ability to cope with labor, a measure of childbirth self efficacy is necessary.
Objectives: This paper aims to assess the cultural adaptation and psychometric testing of the short form of childbirth self-efficacy Inventory among Iranian pregnant women.

Patients and Methods: In this descriptive-methodological study, we investigated 383 Iranian pregnant women in the third trimester They were recruited from the outpatient prenatal care clinic of Taleghani Hospital and an urban health center from August to November 2011. Content validity was evaluated by a panel of specialists after adding two religious items. The women completed the inventory and the demographic characteristics questionnaire in an interview room. The internal consistency and construct validity were assessed by Cronbach's alpha and by exploratory and confirmatory factor analyses, respectively. Known group analysis on gravity assessed the discriminant validity of the measure.

Results: Content validity of the short form of the Iranian childbirth self-efficacy Inventory was confirmed. Factor analyses supported the conceptual two-factor structure of measure and hence supported its construct validity. The internal consistency was approved for the total scale and both subscales. The instrument differentiated prim gravid from multigravida women in the total scale and the efficacy expectancy subscale.

Conclusions: Validity and reliability of the measure supports the use of the short form of the instrument as a clinical and research instrument in measuring childbirth self-efficacy among Iranian pregnant women.
\end{abstract}

Keywords: Childbirth; Self-Efficacy; Pregnancy; Psychometric Testing

\section{Background}

The quality of the perception of childbirth and coping with it, as a developmental event, affects a woman's selfefficacy $(1,2)$. A woman's confidence in her ability plays a major role in coping (3), and self-efficacy towards that is inversely associated with the level of labor pain perception (4). During labor, self-efficacy expectancy would assist a woman to reflect her capabilities in coping with this stressful situation and to perform required behaviors (2). To investigate maternal confidence in coping with labor, Lowe (1993) developed the childbirth self-efficacy inventory (CBSEI) to: 1) promote the conceptual development of delivery confidence and 2) help with effective nursing interventions, (2). Since its development, Outcome Expectancy (OE) and Efficacy Expectancy (EE) have been evaluated by CBSEI in many studies. With a reasonable level of validity and reliability (2, 3, 5-9), the original CBSEI included 62 items, loading in a four factor structure and two repetitive sets of items (10).

However, pregnant women's confidence in their coping behavior showed no differences between the first and second stage of labor in the previous studies $(6,11)$. Since it is too hard to find different responses between two stages of labor based on repetitive and parallel sets of items in pregnant women, it is not recommended to use it for both assessments and there are concerns in this regard. Ip et al. (2008) developed a short-form Chinese CBSEI by deleting two repetitive subscales (OE15 and EE-15) to solve the problem of the repetitive and

Implication for health policy/practice/research/medical education:

This study demonstrates the cultural adoption, validity and reliably of modified short form of Iranian Childbirth Self-Efficacy Inventory (ICBSEI-36) in Iranian pregnant women. It could be used as a measure to perform educational interventions for women needing improve their confidence in coping ability for labor. In addition due to its feasibility and time conserving ability, this short form could be used as the midwives and nursing practicing measure of maternal strength warranting re-enforcement in the clinical settings.

Copyright @ 2013, Kowsar Corp.; Published by Kowsar Corp. This is an open-access article distributed under the terms of the Creative Commons Attribution License, which permits unrestricted use, distribution, and reproduction in any medium, provided the original work is properly cited. 
lengthy structure of the original childbirth self-efficacy inventory. The short form consists of two parallel OE-16 and EE-16 subscales, containing the same 16 items for evaluating childbirth coping behavior (7). The short form CBSEI has adequate predictive validity, construct (both convergence and discriminant) validity, internal consistency and test-retest reliability $(7,10)$. In addition, a study by Khorsandi et al.(2008), they suggested to add religious items to the short form of CBSEI to adapt with the Iranian culture (8), which was considered in this study. Compared to the lengthy structure of the original CBSEI, the short form is that it takes a short time to complete and has not been used in Iranian women. To our knowledge, no such instrument has been used, if any, in Iranian population.

\section{Objectives}

This study was designed to test the cultural compatibility and psychometric properties (including validity and reliably) of the modified short form of Iranian Childbirth Self-Efficacy Inventory (ICBSEI-36) in Iranian pregnant women. The following specific aims were addressed in this study:

1. To determine the adaption of ICBSEI-36 with the added religious items.

2. To determine the factor model for the short form of ICBSEI-36.

3. To determine the discriminability of the responses obtained from the factor structure of the ICBSEI-36 between prim gravid and multigravida women.

4. To determine the internal consistency of factor structure of the ICBSEI-36.

\section{Results}

\subsection{Sample Characteristics}

Out of 400 study cases, 383 returned the questionnaire (Response rate $=95.75 \%$ ). Of them, $68.73 \%$ were primiparous $(n=255)$ and the rest were multiparous $(n=128)$. They were all married and the majority (42.6\%) had high school diploma. Most of the respondents did not attend childbirth education classes (83\%). The Mean maternal age was 32.8 (SD 7.26) years and the mean gestational age was 29.4 weeks (SD 9.4). Mean maternal weight and height were 71.01 (SD 11.7) $\mathrm{kg}$ and 158.62 (SD 19.9) cm, respectively (also for other characteristics see Table 1 ).

The normality of each observed variable based on skewness and kurtosis (due to large sample sizes) was confirmed (absolute skewness $<3$ and absolute kurtosis measure<10) (Table 2).

\subsection{Feasibility}

Ceiling effects were detected for 26 persons (6.8\%) in the OE subscale and for 12 persons (3.1\%) in the EE subscale. There was no Floor effect for theses subscales.

Table 1. Background Characteristics of study participants $(\mathrm{n}=383)^{\mathrm{a}}$

\begin{tabular}{|c|c|}
\hline Characteristics & No.(\%) \\
\hline \multicolumn{2}{|l|}{ Parity } \\
\hline Primiparous & $255(66.6)$ \\
\hline Multiparous & $128(33.4)$ \\
\hline \multicolumn{2}{|l|}{ Educational level } \\
\hline illiterate & $11(2.9)$ \\
\hline Junior high school education & $158(41.5)$ \\
\hline High school diploma & $163(42.8)$ \\
\hline University education & 49 (12.9) \\
\hline \multicolumn{2}{|l|}{ Husband's Educational level } \\
\hline illiterate & $16(4.2)$ \\
\hline Junior high school education & $176(46.7)$ \\
\hline High school diploma & $146(38.7)$ \\
\hline University education & $39(10.3)$ \\
\hline \multicolumn{2}{|l|}{ Occupation } \\
\hline Housewife & $356(93.4)$ \\
\hline Employed in Governmental sector & $13(3.4)$ \\
\hline Self-employed & $12(3.1)$ \\
\hline \multicolumn{2}{|l|}{ Husband's Occupation } \\
\hline Employed in Governmental sector & $51(17.0)$ \\
\hline Self-employed & $236(78.7)$ \\
\hline Unemployed & $13(4.3)$ \\
\hline \multicolumn{2}{|l|}{ Antenatal class attendance } \\
\hline Yes & $318(84.8)$ \\
\hline No & $57(15.2)$ \\
\hline \multicolumn{2}{|l|}{ Insurance Type } \\
\hline social security & $168(48.1)$ \\
\hline Health care & $129(37.0)$ \\
\hline Other & $52(14.9)$ \\
\hline \multicolumn{2}{|l|}{ Household income (monthly) } \\
\hline 100-299 thousand Toman & $140(42.0)$ \\
\hline 300-399 thousand Toman & $162(48.6)$ \\
\hline$=>400$ thousand Toman & $31(9.3)$ \\
\hline
\end{tabular}

$\mathrm{a}$ If the sum of frequency does not match the total number of 383 , there are non-responses in those characteristics. 
Table 2. Summary of statistics of CBSEI-C336 Subscale Scores (n $=383)^{\mathrm{a}}$

\begin{tabular}{lllll}
\hline & Mean & Std. Deviation & Skewness & Kurtosis \\
\hline OE & 134.55 & 32.06 & -0.86 & 0.70 \\
EE & 112.07 & 37.11 & -0.13 & -0.66 \\
Total & 246.62 & 62.85 & -0.49 & 0.17 \\
\hline
\end{tabular}

${ }^{\mathrm{a}}$ The possible range of OE and EE score is $18-180$ and for total score is 36360

\subsection{Content Validity}

In qualitative evaluation of the measure, experts provided written feedback on the clarity and relevancy of the content of the ICBSEI-36 items to the Iranian culture and the content validity of the measure was generally supported. It is noteworthy that some items were revised based on the qualitative suggestions of the panel experts.

\subsection{Reliability}

The Cronbach's alpha was 0.92 for the total scale and
0.88 and 0.88 for the subscales measuring OE and EE respectively, indicating adequate internal consistency (>0.7). Guttman's split half index for total scale (0.78), OE subscale (0.82) and EE subscale (0.83) showed a satisfactory $(>0.7)$ split half reliability.

\subsection{Construct Validity}

For evaluating construct validity, both EFA and CFA were performed for the items of OE and EE subscales.

\subsubsection{EFA}

In this analysis, KMO measures of sampling adequacy were 0.902 and 0.903 for $\mathrm{OE}$ and EE respectively, which supported the sampling adequacy of the data for modeling for these subscales. Bartlett's test of sphericity gave $\mathrm{P}<0.05$. The Scree plot for both subscales supported the uni-dimensionality of each one.

Cut-off values $\geq 0.3$ for factor loadings showed that all items related to $\mathrm{OE}$ and EE subscales were reasonably loaded on theses subscales (Table 3). The results suggested that each ICBSEI-36 subscale was one-dimensional.

Table 3. Factor Loadings for OE and EE Subscales

\begin{tabular}{|c|c|c|c|}
\hline Items of $\mathrm{OE}$ & Factor Loadings & Items of EE & Factor Loadings \\
\hline 9. Stay on top of each contraction & 0.653 & 4. Keep myself in control & 0.715 \\
\hline 8. Concentrate on thinking about the baby & 0.642 & 9. Stay on top of each contraction & 0.712 \\
\hline 10. Think positively & 0.634 & 10. Think positively & 0.690 \\
\hline 12. Tell myself that I can do it & 0.628 & 2. Get ready for each contraction & 0.686 \\
\hline 16. Focus on the person helping me in labour & 0.624 & 12. Tell myself that I can do it & 0.684 \\
\hline $\begin{array}{l}\text { 15. Listen to encouragement from the person } \\
\text { helping me }\end{array}$ & 0.614 & 7. Keep myself calm & 0.669 \\
\hline 7. Keep myself calm & 0.567 & 5. Think about relaxing & 0.641 \\
\hline 5. Think about relaxing & 0.552 & 11. Not think about the pain & 0.581 \\
\hline $\begin{array}{l}\text { 14. Concentrate on getting through one con- } \\
\text { traction at a time }\end{array}$ & 0.528 & $\begin{array}{l}\text { 6. Concentrate on an object in the room } \\
\text { to distract myself }\end{array}$ & 0.570 \\
\hline 3. Use breathing during labour contractions & 0.517 & $\begin{array}{l}\text { 8. Concentrate on thinking about the } \\
\text { baby }\end{array}$ & 0.567 \\
\hline 17. I Praise God and ask for help from him & 0.514 & $\begin{array}{l}\text { 3. Use breathing during labour contrac- } \\
\text { tions }\end{array}$ & 0.563 \\
\hline 11. Not think about the pain & 0.504 & 1. Relax my body & 0.561 \\
\hline 2. Get ready for each contraction & 0.492 & 13. Think about others in my family & 0.558 \\
\hline 1. Relax my body & 0.488 & $\begin{array}{l}\text { 16. Focus on the person helping me in } \\
\text { labour }\end{array}$ & 0.539 \\
\hline 13. Think about others in my family & 0.465 & $\begin{array}{l}\text { 14. Concentrate on getting through one } \\
\text { contraction at a time }\end{array}$ & 0.538 \\
\hline $\begin{array}{l}\text { 6. Concentrate on an object in the room to } \\
\text { distract myself }\end{array}$ & 0.460 & 18. Walking between labour pain & 0.444 \\
\hline 4. Keep myself in control & 0.407 & 17. I Praise God and ask for help from him & 0.350 \\
\hline 18. Walking between labour pain & 0.399 & $\begin{array}{l}\text { 15. Listen to encouragement from the } \\
\text { person helping me }\end{array}$ & 0.312 \\
\hline
\end{tabular}




\subsubsection{CFA}

The model showed reasonably good fit indices (x2/degrees of freedom $(\mathrm{df})=3.70<5 ;$ SRMR $=0.07<0.1$, RMSEA $=0.000<0.08((90 \%$ confidence interval $(\mathrm{CI}))=(0.000$; $0.010)), \mathrm{CFI}=0.99>0.90, \mathrm{NFI}=1.00>0.90, \mathrm{NNFI}=1.01>$ 0.90 and good support for the two-factor structure of the ICBSEI-36 (MacCallum, Browne, \& Sugawara, 1996; Kline, 2005; Marsh, Balla, \& Hau, 1996).

Based on this model, factor loadings indicate significant loadings on the two-factor solution (Figure 2). Standardized factor loadings ranged from 0.41 to 0.64 on the $\mathrm{OE}$ subscale, and from 0.38 to 0.71 on the EE subscale, with all items demonstrating moderate to strong factor loadings (above 0.30)( 21 ) (Figure 1).

The statistical significance of the two-factor correlations $(\mathrm{r}=0.70, \mathrm{P}<.01)$ supported the hypothesis that the two factors (OE and EE subscales) were highly related dimensions of childbirth self-efficacy.

\subsection{Convergent Validity}

The correlation coefficient between the subscales and the total scale was $0.89(\mathrm{P}<0.01)$ for the OE subscale and
$0.92(\mathrm{P}<.01)$ for the EE subscale, and the correlation coefficient between the subscales was $0.65(\mathrm{P}<.01)$, indicating a significant overlap between the two subscales.

\subsection{Known Group Analysis}

To assess the discriminant validity of the scales, the results of multivariate test for comparing multigravid and primigraid women showed a significant simultaneous difference (Hotteling T2 $=0.025, \mathrm{~F}(2,368)=4.64$ and $\mathrm{P}=0.010$ ). Also, univariate analysis showed a significant difference between primi an multigravid women in the total scale $(\mathrm{t}=-2.118, \mathrm{df}=369$ and $\mathrm{P}=0.035)$ and the EE subscale $(\mathrm{t}=-2.811, \mathrm{df}=369$ and $\mathrm{P}=0.005)$ with a higher mean score for multigravid women (Table 4), providing evidence supportive for the discriminant (construct) validity of the ICBSEI-36 as indicated by known group procedure. However, for the OE subscale, the result was insignificant $(\mathrm{t}=-0.884$, $\mathrm{df}=369$ and $\mathrm{P}$ $=0.377$ ).

Figure 1. CFA Factor Loadings for OE and EE Subscales

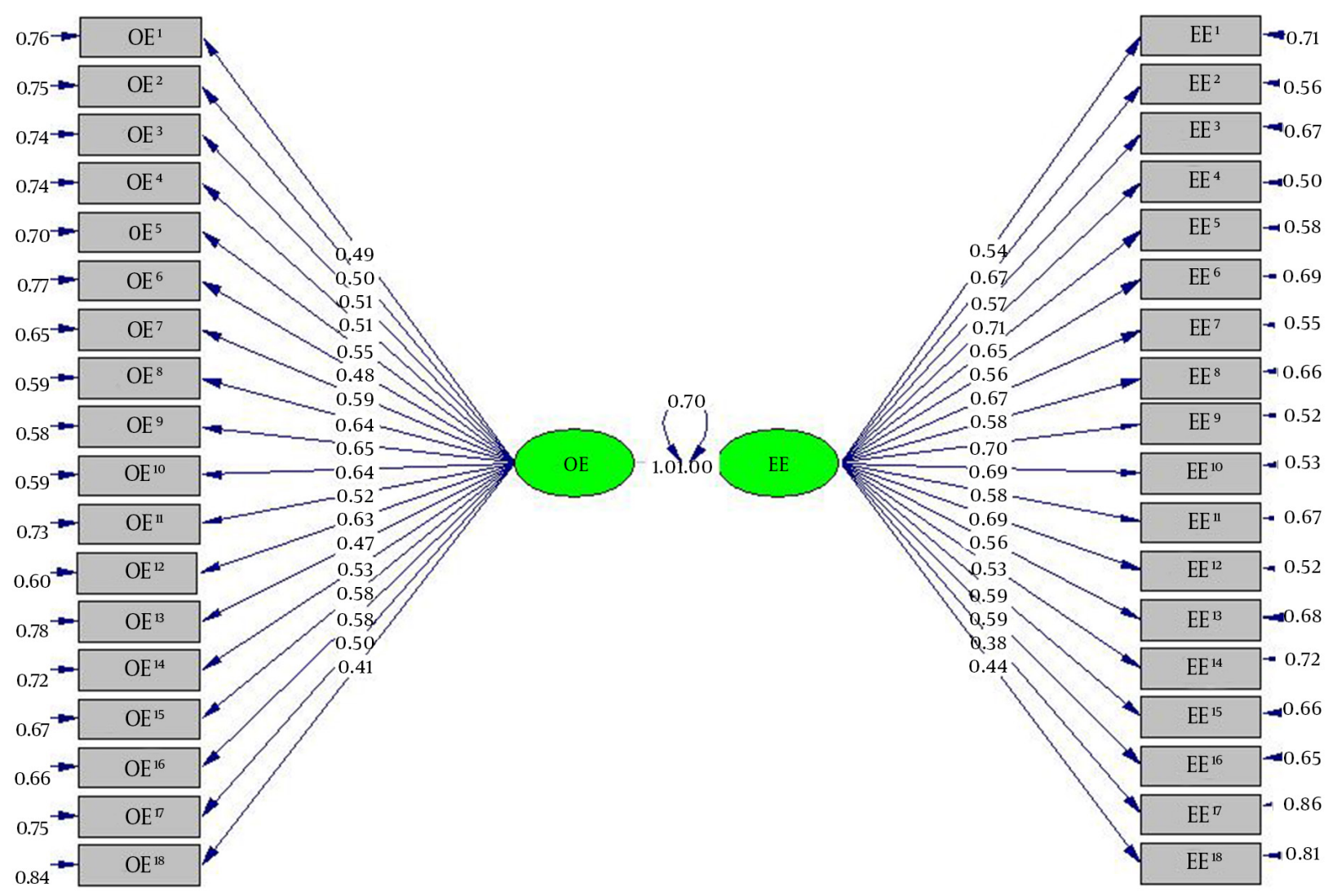

All factor loadings were statistically significant (All $\mathrm{P}<0.05$ ) and there was a significant correlation between $\mathrm{OE}$ and $\mathrm{EE}$ subscales $(\mathrm{P}<0.05)$. 
Khorsandi M et al.

\begin{tabular}{|c|c|c|c|c|c|c|}
\hline PMGravid & No & Mean & Std. Deviation & $\mathbf{t}^{\mathrm{b}}$ & df & Sig. (2-tailed) \\
\hline \multicolumn{7}{|l|}{$\mathbf{O E}$} \\
\hline Primigravid & 255 & 134.54 & 32.40 & -0.88 & 369 & 0.377 \\
\hline Multigravid & 116 & 137.66 & 29.69 & & & \\
\hline \multicolumn{7}{|l|}{$\mathbf{E E}$} \\
\hline Primigravid & 255 & 109.00 & 35.83 & -2.81 & 369 & 0.005 \\
\hline Multigravid & 116 & 120.50 & 38.00 & & & \\
\hline \multicolumn{7}{|l|}{ Total } \\
\hline Primigravid & 255 & 243.54 & 60.66 & -2.12 & 369 & 0.035 \\
\hline Multigravid & 116 & 258.16 & 63.83 & & & \\
\hline
\end{tabular}

\section{Discussion}

Findings provided support for acceptable reliability and validity of ICBSEI-36 for the assessment of childbirth self-efficacy among pregnant women in Iran. In a study conducted in a sample of the Chinese population in Hong Kong, the measure showed a reasonable level of validity and reliability as a self-report measure of women's childbirth self-efficacy $(7,10)$.

\subsection{Feasibility}

Ceiling effects of $6.8 \%$ and $3.1 \%$ were detected for OE and EE subscales respectively but there was no floor effect for theses subscales, which confirmed the feasibility of the measure in the Iranian population. No studies related to this instrument present measure of ceiling and floor effects.

\subsection{Content Validity}

The content validity of ICBSEI-36 was supported based on the evaluation of a panel of experts. The same procedure was performed and the same results were achieved in a study conducted by Gallo et al. (2011) (10).

\subsection{Reliability}

The ICBSEI-36 had acceptable internal consistency (Cronbach's alpha fulfilling the criteria), indicating a satisfactory degree of consistency among items for each subscale. In other studies, high internal consistency reliability has been reported for the original measure (0.82$0.96)(2,5,6)$ and also for the short form of the measure $(7,10)$.

\subsection{Construct Validity}

The results of EFA and CFA provided evidence the unidmentionality structure for each subscale of the ICBSEII36, reflecting the consistency of the two dimensions of
OE and EE subscales with the original factor structure identified in previous researches (7). The results of other studies also suggest that each ICBSEI-I36 subscale is onedimensional, leading in a two-factor structure $(2,5,6,10)$.

\subsection{Convergent Validity}

High values of the correlation between subscales, which indicated a significant overlap between the two subscales, supported the convergent validity of the measure. Similarly, the convergent validity with the Chinese self-efficacy scale was reflected by a moderate correlation for the two subscales (7).

\subsection{Discriminant Validity}

The parity differences observed for the ICBSEIC-36, with higher subscale and scale scores for multigravid versus primigravid women in EE, were consistent with the theoretical construct of the measure (19). According to Bandura (1997), direct experiencing of any event, such as childbirth, affects the perceptions of efficacy beliefs, as a powerful source of information (19). Women with positive previous experiences in labor are more probable to have a higher perceived self-efficacy for a forthcoming birth and to report a positive childbirth experience (22). This results were in line with those reported by Ip et al. (2008) and Lowe (1993) in which EE scores differentiated primigravid women from multigravida women $(2,7)$. However, Gao et al (2011) observed higher scores of both OE-16 and EE-16 subscales in multigravid than primigravid women (10); hence, further research is needed to explore the discriminant validity of the OE scale.

The strength of this study includes a large sample size, fulfilling the requirement of 5 or more participants per item for factor analysis. However, most samples were well educated, married, and from middle-class society; thus, generalizability of the results is limited. In addition, it is recommended to perform CFA in a different sample. 
This study showed that the ICBSEI-36 was a valid, reliable and culturally compatible measure which could be used as a research instrument. Also, it was found to be short and feasible enough to be used as a clinical instrument for measuring childbirth self-efficacy in Iranian pregnant women. It could be employed as a measure to perform educational interventions in women who need to improve their confidence in coping ability for labor. In addition, due to its feasibility and time conserving nature, this short form could be used by midwives, nurses and clinicians who are involved in the care of pregnant mothers in order to identify mothers who need psychological support.

\section{Acknowledgements}

We are grateful to the Research Deputy of Arak University of Medical Sciences for supporting the study.

\section{Author Contributions}

Study concept and design: Khorsandi and Rafiei. Data Collection: Jahani, Analysis and interpretation of data: Asghari Jafarabadi. Drafting of the manuscript: Khorsandi and Asghari Jafarabadi. Critical revision of the manuscript for important intellectual content: Khorsandi and Asghari Jafarabadi. Statistical analysis: Asghari Jafarabadi.

\section{Financial Disclosure}

No conflict of interest has been declared by the authors and all of them have no financial interests related to the material in the manuscript.

\section{Funding/Support}

Research deputy of Arak University of Medical Sciences for financial supporter of the study.

\section{References}

1. Ip WY, Tang CS, Goggins WB. An educational intervention to improve women's ability to cope with childbirth. J Clin Nurs 2009;18(15):2125-35.

2. Lowe NK. Maternal confidence for labor: development of the Childbirth Self-Efficacy Inventory. Res Nurs Health. 1993;16(2):141-
3. Sinclair M, O'Boyle C. The Childbirth Self-Efficacy Inventory: a replication study. J Adv Nurs. 1999;30(6):1416-23.

4. Sieber S, Germann N, Barbir A, Ehlert U. Emotional well-being and predictors of birth-anxiety, self-efficacy, and psychosocial adaptation in healthy pregnant women. Acta Obstet Gynecol Scand. 2006;85(10):1200-7.

5. Cunqueiro MJ, Comeche MI, Docampo D. Childbirth Self-Efficacy Inventory: psychometric testing of the Spanish version. J Adv Nurs. 2009;65(12):2710-8.

6. Drummond J, Rickwood D. Childbirth confidence: validating the Childbirth Self-Efficacy Inventory (CBSEI) in an Australian sample. J Adv Nurs. 1997;26(3):613-22.

7. Ip WY, Chung TK, Tang CS. The Chinese Childbirth Self-Efficacy Inventory: the development of a short form. J Clin Nurs. 2008;17(3):333-40.

8. Khorsandi M, Ghofranipour F, Faghihzadeh S, Hidarnia A, Bagheban AA, Aguilar-Vafaie ME. Iranian version of childbirth selfefficacy inventory. J Clin Nurs. 2008;17(21):2846-55.

9. Tanglakmankhong K, Perrin NA, Lowe NK. Childbirth Self Efficacy Inventory and Childbirth Attitudes Questionnaire: psychometric properties of Thai language versions. J Adv Nurs 2011;67(1):193-203.

10. Gao LL, Ip WY, Sun K. Validation of the short form of the Chinese Childbirth Self-Efficacy Inventory in Mainland China. Res Nurs Health. 2011;34(1):49-59.

11. Ip WY, Chan D, Chien WT. Chinese version of the Childbirth Selfefficacy Inven tory. J Adv Nurs. 2005;51(6):625-33.

12. Bryant FB, Yarnold PR. Principal components analysis and exploratory and confirmatory factor analysis. In: Grimm LG, Yarnold PR editors. Reading and Understanding Multivariate Statistics.: American Psychological Association; 1998.

13. DeVellis RF. Scale Development: Theory and Applications. 2nd edThousand Oaks, CA: SAGE Publications; 2003.

14. Chou CP, Bentler PM. Estimates and tests in structural equation modeling. In: Hoyle RH editor. Structural Equation Modeling: Concepts, Issues, and Applications. Thousand Oaks, CA: SAGE Publications; 1995.

15. Klein DF. Review of Beyond significance testing: Reforming data analysis methods in behavioral research. Am J Psychiat. 2005;162

16. McHorney CA, Tarlov AR. Individual-patient monitoring in clinical practice: are available health status surveys adequate? Quality of Life Research.1995;4(4):293-307.

17. Tinsley HEA, Brown SD. Handbook of Applied Multivariate Statistics and Mathematical Modeling.: Elsevier Science; 2000.

18. Kline P. An Easy Guide to Factor Analysis.: Routledge; 1994

19. Bandura A. . Self-efficacy and health behaviour. In: Baum A, New man S, Wienman J, West R, McManus C editors. Cambridge Handbook of Psychology, Health and Medicine.: Cambridge University Press; 1997.

20. Bandura A. Self-efficacy. The Corsini Encyclopedia of Psychology. 4th ed. Hoboken, New Jersey: John Wiley \& Sons; 2010. p. 1534-1536.

21. Cohen J. Statistical power analysis for the behavioral sciences. 2nd ed. Hillsdale, NJ: Erlbaum; 1988.

22. Slade P, MacPherson SA, Hume A, Maresh M. Expectations, experiences and satisfaction with labour. Br J Clin Psychol. 1993;32 ( Pt 4):469-83. 